\title{
A kinetic model for redox-active film based biophotoelectrodes $\uparrow$
}

\author{
D. Buesen, (D) T. Hoefer, (D) H. Zhang (D) and N. Plumeré (iD *
}

Received 5th November 2018, Accepted 18th December 2018

DOI: $10.1039 / c 8 f d 00168 \mathrm{e}$

Redox-active films are advantageous matrices for the immobilization of photosynthetic proteins, due to their ability to mediate electron transfer as well as to achieve high catalyst loading on an electrode for efficient generation of electricity or solar fuels. A general challenge arises from various charge recombination pathways along the lightinduced electron transfer chain from the electrode to the charge carriers for electricity production or to the final electron acceptors for solar fuel formation. Experimental methods based on current measurement or product quantification are often unable to discern between the contributions from the photocatalytic process and the detrimental effect of the short-circuiting reactions. Here we report on a general electrochemical model of the reaction-diffusion processes to identify and quantify the "bottlenecks" present in the fuel or current generation. The model is able to predict photocurrenttime curves including deconvolution of the recombination contributions, and to visualize the corresponding time dependent concentration profiles of the product. Dimensionless groups are developed for straightforward identification of the limiting processes. The importance of the model for quantitative understanding of biophotoelectrochemical processes is highlighted with an example of simulation results predicting the effect of the diffusion coefficient of the charge carrier on photocurrent generation for different charge recombination kinetics.

\section{Introduction}

Photosynthetic proteins have evolved toward having near perfect light harvesting and charge separation properties, which makes them potentially valuable as photoactive components in devices for conversion of sunlight into electricity or solar fuels. ${ }^{1-3}$ A variety of biophotocathodes has been reported which typically follow the same general design. A natural or artificial electron mediator is used to shuttle electrons between the electrodes and the donor side $\left(\mathrm{D} / \mathrm{D}^{+}\right)$of the photosynthetic protein. An electron acceptor then recovers the electron at the acceptor site $\left(\mathrm{A} / \mathrm{A}^{+}\right)$of the protein which can subsequently act either (i) as a charge

Center for Electrochemical Sciences (CES), Faculty of Chemistry and Biochemistry, Ruhr University Bochum, Universitätsstr. 150, D-44780 Bochum, Germany. E-mail: nicolas.plumere@rub.de

$\dagger$ Electronic supplementary information (ESI) available. See DOI: 10.1039/c8fd00168e 
carrier that diffuses to the collector electrode to close the electrical circuit and generate electricity or (ii) as a redox catalyst that generates chemical energy in the form of a solar fuel in a follow up coupled reaction (Fig. 1).

In both cases, the overall energy conversion efficiency is closely related to the rates of electron transport defining the photocurrent and to the redox potential of the various components defining the light-induced potential difference within the electrochemical half-cell and thus the potential energy gain. From a practical perspective, it is advantageous to immobilize the electron mediator and the photosynthetic proteins within thin redox films on the electrode surface to allow for efficient electrical wiring and for high catalyst loading and thus obtain high photocurrent generation.,

However, besides the photocatalytic process, possible competitive pathways may have a detrimental impact on the performance of such biophotocathodes. One of the general challenges in photoelectrochemical systems is related to charge recombination processes. ${ }^{6}$ Light-induced charge separation at the photosystem produces a high energy electron that is ideally transferred to the charge carriers or to the redox catalyst with minimal energy loss. However, the large driving force imposed by the light-induced potential difference favors recombination of these reduced electron acceptors with oxidized components of the redox matrix or with the electrode surface (Fig. 1, red pathway). ${ }^{7,8}$ These shortcircuiting processes lower the photocurrents and hence the overall power output or solar fuel generation of the devices. Therefore, in-depth understanding of the processes involved in photocurrent generation, including such short circuit pathways, is an essential pre-requisite for the rational design and optimization of biophotoelectrochemical systems.

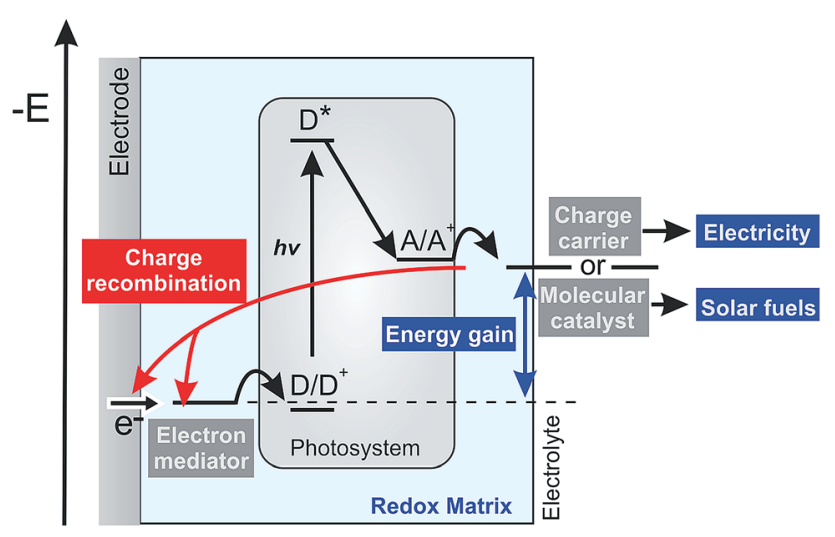

Fig. 1 Energy level diagram and schematic illustration of reactions in a biophotoelectrode based on photosynthetic proteins. The light-induced charge separation at the photosynthetic protein triggers the electron transfer chain. The electron mediator refills the hole at the donor site $\left(\mathrm{D} / \mathrm{D}^{+}\right)$of the photosystem and a charge carrier or a molecular catalyst recovers the electron from the acceptor site $\left(A / A^{+}\right)$. The electron mediator shuttles the charge from the electrode while the charge carrier can be used to generate electricity or the molecular redox catalyst catalyzes reactions for solar fuel generation. The energy gained from light leads to a potential difference between the electron mediator and the charge carrier defines the driving force for charge recombination of the charge carrier at the mediator or at the electrode. 
The short-circuiting processes are often invisible to electroanalytical methods since their contribution do not provide any net photocurrent. Therefore, it is paramount to establish theoretical models for biophotoelectrochemical systems which consider both the processes generating the photocurrent and the processes competing with photocurrent generation. The ability to simulate and deconvolute the various contributions, including photocathodic processes and recombination processes, would enable the pinpointing of bottlenecks in the power generation process. Ideally, such a model would not only include simulations for the entire observed signal, but would also contain the development of dimensionless groups which are useful for summarizing the rates of the major processes in the system, and in particular, predict how a given parameter may impact photocurrents and thus how it could be modulated to achieve energy conversion enhancement.

Several models have been previously developed for biophotoelectrochemical systems, which considered photosynthetic proteins ${ }^{9-12}$ or whole photosynthetic cells immobilized on electrodes or in solution. ${ }^{13,14}$ In these previous reports, electronic communication between the photosystems and the electrode were modelled based on freely diffusing electron mediators. Here, we establish a model for biophotoelectrodes with both the photosystems and the electron mediators confined in redox films on the electrode surface based on a reaction scheme that is generally applicable and relevant for multiple experimental cases. ${ }^{\mathbf{1 , 4 1 5 , 1 6}}$ In particular, we consider both an outer-sphere electron transfer between the photosystem and the electron acceptor (which is typically relevant for photosystem 1 based biophotocathodes) as well as photoenzymatic reactions (which are typically relevant for biophotocathodes based on purple bacterial reaction centers). Moreover, we include the possibility for either electron transfer to a charge carrier that subsequently diffuses to the bulk of the solution or for electron transfer to a redox catalyst followed by subsequent catalytic reduction of a final electron acceptor generating solar fuels. The model is built upon previous models for bioelectrochemical systems ${ }^{17,18}$ in which we integrate the effect of light induction of electron transfer and the associated charge recombination processes to predict the time dependent photocurrent generation and the associated concentration profile. Dimensionless groups are developed for understanding the limiting processes. We highlight the usefulness of this modeling tool with an example of simulation results predicting the effect of the diffusion coefficient of the charge carrier on photocurrent generation with different charge recombination kinetics.

\section{System schematic and reactions}

The process generating the photocurrent (Fig. 2, in black) includes the redox mediator $M_{\text {red }} \mid M_{\text {ox }}$ and the photosystem $P_{\text {red }} \mid P_{\text {ox }}$ which are immobilized in the redox film, as well as the electron acceptors $Y_{\mathrm{ox}}$ and $Z_{\mathrm{ox}}$ and their respective reduced forms $Y_{\text {red }}$ and $Z_{\text {red }}$ which are freely diffusing within the film and in the bulk of the solution. The charge hopping between the redox mediators is assumed to follow the rules of diffusion and is described by an apparent diffusion coefficient $D_{\mathrm{M}}$. The partition of both substrate-product pairs $Y_{\text {red }} \mid Y_{\mathrm{ox}}$ and $Z_{\text {red }} \mid Z_{\text {ox }}$ is neglected and their diffusion coefficients $\left(D_{\mathrm{Y}}\right.$ and $D_{\mathrm{Z}}$, respectively) remain unchanged whether they are in or out of the redox matrix. 


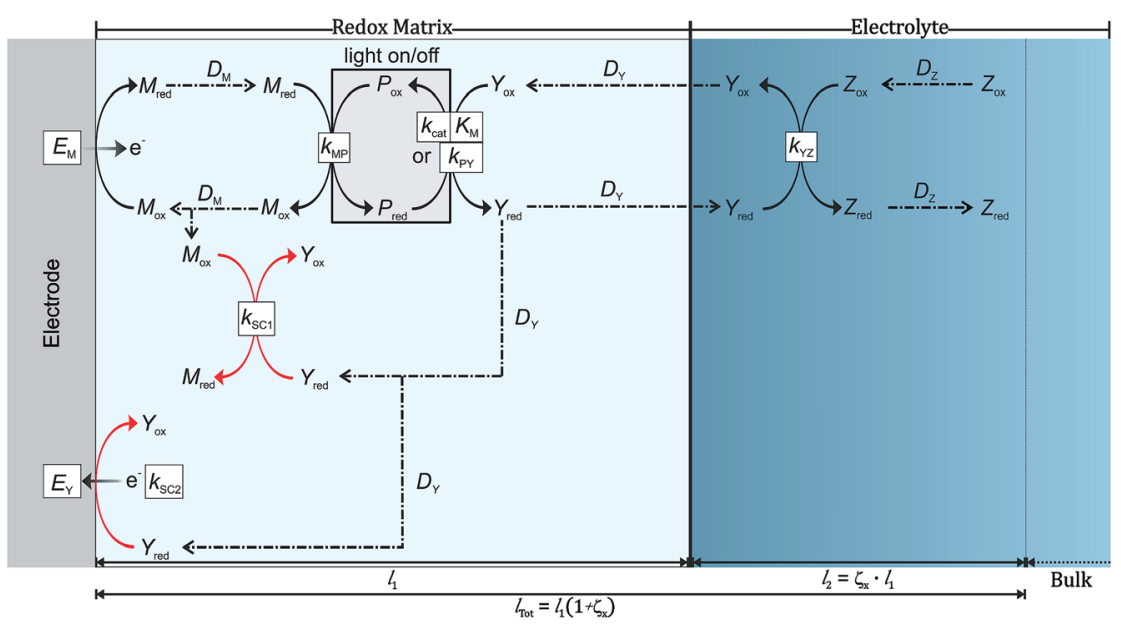

Fig. 2 Schematic illustration of electron transfer pathways for a biophotocathode based on photosynthetic proteins immobilized within a redox film containing electron mediators for shuttling electrons between the protein and the electrode. The process contributing to photocurrent generation is given in black while the short-circuiting processes are given in red.

Furthermore, we assume a steady state between the oxidized and reduced form of the photosystems while catalysis is taking place. The model considers only one electrochemical half-cell in which a constant potential is applied at the electrode. The heterogeneous electron transfer kinetics of $M_{\text {red }} \mid M_{\text {ox }}$ are modeled according to Butler-Volmer kinetics. $M_{\text {red }}$ and $P_{\text {ox }}$ react in a bimolecular reaction with a rate constant $k_{\mathrm{MP}}$ in single one-electron steps. This reaction is followed by a lightinduced reaction between the reduced form of the photosystem with $Y_{\mathrm{ox}}$, which is either modeled according to Michaelis-Menten kinetics $\left(k_{\text {cat }}\right.$ and $\left.K_{\mathrm{M}}\right)$ or by a simple bimolecular reaction $\left(k_{\mathrm{PY}}\right)$. This reaction leads to $Y_{\text {red }}$ which may serve as a charge carrier that diffuses in the bulk of the solution or as a redox catalyst that reduces another electron acceptor $Z_{\mathrm{ox}}$ in a subsequent reaction cascade leading to $Z_{\text {red }}$. In the latter case $Y_{\text {red }}$ reacts in a bimolecular reaction with $Z_{\text {ox }}$ with a kinetic constant $k_{\mathrm{YZ}}$. Because both $Y_{\text {red }}$ and $Z_{\mathrm{ox}}$ are free to diffuse in and out of the film this reaction can take place either in the redox matrix or in the surrounding electrolyte solution. The produced solar fuel $Z_{\text {red }}$ can then diffuse into the bulk of the system.

In order to consider the possibility for charge recombination we consider two short-circuiting reactions involving $Y_{\text {red }}$ (Fig. 2, in red). The redox potential of $Y_{\text {red }} \mid Y_{\mathrm{ox}}\left(E_{\mathrm{Y}}^{0}\right)$ is more negative than the redox potential of $M_{\text {red }} \mid M_{\mathrm{ox}}$ $\left(E_{\mathrm{M}}^{0}\right)$. Therefore, the potential difference favors the reduction of $M_{\mathrm{ox}}$ by $Y_{\text {red }}$. We model this first short-circuiting pathway (SC1) as a bimolecular reaction with a kinetic constant $k_{\mathrm{SC} 1}$. The second possible short-circuiting pathway (SC2) takes place at the electrode and leads to the reoxidation of $Y_{\text {red }}$. The heterogeneous (multi-) electron transfer is also modeled according to Butler-Volmer kinetics with a heterogeneous rate constant $k_{\mathrm{SC} 2}^{0}$ and an apparent electron transfer coefficient $\alpha_{\mathrm{Y}}$.

The reaction stoichiometry between $Y_{\mathrm{ox}}$ and $P_{\text {red }}$ is fixed as one-to-one, but the number of electrons transferred in the reaction $\left(\nu_{\mathrm{Y}}\right)$ is variable. Additionally, the 
number of electrons that are transferred from $P_{\mathrm{ox}}$ to $M_{\mathrm{red}}\left(\nu_{\mathrm{M}}\right)$ is flexible depending on the properties of the mediator. The number of electrons transferred between $Y_{\text {red }}$ and $M_{\mathrm{Ox}}$ in the SC1 process depends on the ratio of $\nu_{\mathrm{Y}}$ and $\nu_{\mathrm{M}}$.

\section{Modeling equations}

\subsection{Space and time domains}

The total space $\left(l_{\text {tot }}\right)$ is divided into two space domains: (1) the redox film, and (2) the stationary surrounding solution. As shown in eqn (1), the length of the film domain is $l_{1}$, and the length of the second domain has a length which is set as a multiple of the film thickness $\left(\zeta_{\mathrm{x}}\right)$.

$$
l_{\mathrm{tot}}=l_{1}+l_{1} \zeta_{\mathrm{x}}=l_{1}\left(1+\zeta_{\mathrm{x}}\right)
$$

As shown in eqn (2), the total time $\left(t_{\text {tot }}\right)$ is divided into three time domains: (1) when the light is initially off $\left(t_{\mathrm{eq}}\right),(2)$ when the film is under photoillumination $\left(t_{\text {exp }}\right)$, and (3) when the light is off once again $\left(t_{\text {rec }}\right)$.

$$
t_{\mathrm{tot}}=t_{\mathrm{eq}}+t_{\mathrm{exp}}+t_{\mathrm{rec}}=\tau_{\mathrm{tot}} t_{\mathrm{exp}}
$$

By means of the factor $\tau_{\text {tot }}$, the total time is expressed as a multiple of the exposure time, which is the primary time related variable of interest. Information regarding the calculation of $\tau_{\text {tot }}$ is given in the ESI. $\dagger$

\subsection{Main equations}

The main modeling equations were derived from material balances on the reduced form of the mediator $\left(M_{\text {red }}\right)$, on the oxidized form of the first electron acceptor $\left(Y_{\mathrm{ox}}\right)$, and on the oxidized form of the final electron acceptor $\left(Z_{\mathrm{ox}}\right)$. Equations for these species in the film domain are shown in eqn (3)-(5) respectively. They constitute a system of coupled partial differential equations (PDEs) with time, space, and concentration as the primary variables. The equations account for transient behavior by the presence of first order time derivatives. They also account for 1-dimensional spatial variation related to diffusion by the presence of second order spatial derivatives. These are considered within the context of several interrelated chemical and electron transfer reactions. The equations were scaled with respect to their maximum possible values, so that the range of the major dimensionless variables (time, space, and concentrations) is between 0 and 1 .

Reaction stoichiometry is explicitly included in the model, and allows for flexibility with regards to the number of electrons that can be transferred to the electrode by the mediator, and by the first electron acceptor $\left(Y_{\text {red }} \mid Y_{\text {ox }}\right)$, which appear as $z_{\mathrm{M}}$ and as $z_{\mathrm{Y}}$ in the modeling equations, respectively. The stoichiometry between the first electron acceptor and the second electron acceptor $\left(Z_{\text {red }} \mid Z_{\text {ox }}\right)$ is fixed as one-to-one.

These modeling equations shown for the film domain represent the most complex form of these equations, and the corresponding equations for simpler reaction schemes, or for the main equations in the solution domain can be deduced by setting the relevant kinetic terms or concentrations to zero. For example, the main equation for $Y_{\mathrm{ox}}$ in the solution domain can be deduced by setting $\kappa_{\mathrm{CAT}}^{\mathrm{Y}}$ and $\kappa_{\mathrm{SC} 1}^{\mathrm{Y}}$ in eqn (4) to zero. 


$$
\begin{aligned}
{\left[\frac{\omega_{\mathrm{M}}}{\tau_{\mathrm{tot}}}\right] \frac{\partial M_{\mathrm{red}}}{\partial t}=} & {\left[\frac{1}{\left(1+\zeta_{\mathrm{x}}\right)^{2}}\right] \frac{\partial^{2} M_{\mathrm{red}}}{\partial x^{2}}-\frac{\left(\nu_{\mathrm{M}}\right) \kappa_{\mathrm{cat}}^{\mathrm{M}} M_{\mathrm{red}} Y_{\mathrm{ox}}}{\left(\frac{1}{\nu_{\mathrm{M}}}\right) \mu M_{\mathrm{red}}+\left(\frac{1}{\nu_{\mathrm{M}}}\right) M_{\mathrm{red}} Y_{\mathrm{ox}}+\theta_{\mathrm{MM}} Y_{\mathrm{ox}}} } \\
& -\frac{\left(\nu_{\mathrm{M}}\right) \kappa_{\mathrm{MP}}^{\mathrm{M}} M_{\mathrm{red}} Y_{\mathrm{ox}}}{\left(\frac{1}{\nu_{\mathrm{M}}}\right) M_{\mathrm{red}}+\theta_{\mathrm{MP}} Y_{\mathrm{ox}}}+\left(\frac{\nu_{\mathrm{M}}}{\nu_{\mathrm{Y}}}\right) \kappa_{\mathrm{SC} 1}^{\mathrm{M}}\left(1-M_{\mathrm{red}}\right)\left(1-Y_{\mathrm{ox}}\right) \\
{\left[\frac{\omega_{\mathrm{Y}}}{\tau_{\mathrm{tot}}}\right] \frac{\partial Y_{\mathrm{ox}}}{\partial t}=} & {\left[\frac{1}{\left(1+\zeta_{\mathrm{x}}\right)^{2}}\right] \frac{\partial^{2} Y_{\mathrm{ox}}}{\partial x^{2}}-\frac{1}{\left(\frac{1}{\nu_{\mathrm{M}}}\right) \mu M_{\mathrm{red}}+\left(\frac{1}{\nu_{\mathrm{M}}}\right) M_{\mathrm{red}} Y_{\mathrm{ox}}} } \\
& -\frac{\left(\frac{1}{\nu_{\mathrm{Mx}}}\right) \kappa_{\mathrm{MM}} Y_{\mathrm{ox}}}{\left(\frac{1}{\nu_{\mathrm{M}}}\right)\left(\theta_{\mathrm{MP}}\right)^{-1} M_{\mathrm{red}}+Y_{\mathrm{ox}}}+\left(\frac{\nu_{\mathrm{Y}}}{\nu_{\mathrm{M}}}\right) \kappa_{\mathrm{SCl}}^{\mathrm{Y}}\left(1-M_{\mathrm{red}}\right)\left(1-Y_{\mathrm{ox}}\right) \\
& +\kappa_{\mathrm{YZ}}^{\mathrm{Y}} Z_{\mathrm{ox}}\left(1-Y_{\mathrm{ox}}\right) \\
& {\left[\frac{\omega_{\mathrm{Z}}}{\tau_{\mathrm{tot}}}\right] \frac{\partial Z_{\mathrm{ox}}}{\partial t}=\left[\frac{1}{\left(1+\zeta_{x}\right)^{2}}\right] \frac{\partial^{2} Z_{\mathrm{ox}}}{\partial x^{2}}-\kappa_{\mathrm{YZ}}^{\mathrm{Y}} Z_{\mathrm{ox}}\left(1-Y_{\mathrm{ox}}\right) }
\end{aligned}
$$

\subsection{Contributions to current}

The total current is the sum of two contributions, as shown in eqn (6). The photocatalytic contribution to the current $\left(\iota_{\text {cat }}\right)$ is calculated based on the concentration gradient of the reduced form of the mediator at the electrode surface as shown in eqn (7). The SC2 contribution to the current $\left(\iota_{\mathrm{SC} 2}\right)$ is calculated based on the concentration gradient of the oxidized form of the first electron acceptor $Y_{\mathrm{ox}}$ at the electrode surface, as shown in eqn (8).

$$
\begin{gathered}
\iota_{\mathrm{tot}}=\iota_{\mathrm{cat}}+\iota_{\mathrm{SC} 2} \\
\iota_{\mathrm{cat}}=\frac{1}{\left(1+\zeta_{\mathrm{x}}\right)}\left(\frac{\partial M_{\mathrm{red}}}{\partial x}\right)_{x=0}=\sigma_{\mathrm{b}}^{\mathrm{M}}\left(M_{\mathrm{red}}\right)_{x=0}-\sigma_{\mathrm{f}}^{\mathrm{M}}\left[1-\left(M_{\mathrm{red}}\right)_{x=0}\right] \\
\iota_{\mathrm{SC} 2}=\frac{1}{\left(1+\zeta_{\mathrm{x}}\right)}\left(\frac{\partial Y_{\mathrm{ox}}}{\partial x}\right)_{x=0}=\sigma_{\mathrm{b}}^{\mathrm{Y}}\left[1-\left(Y_{\mathrm{ox}}\right)_{x=0}\right]-\sigma_{\mathrm{f}}^{\mathrm{Y}}\left(Y_{\mathrm{ox}}\right)_{x=0}
\end{gathered}
$$

In contrast to the SC2 recombination process, current loss due to the SC1 recombination process is manifested indirectly as a reduction in the main current. In order to isolate the effect of SC1 recombination, each simulation is run twice: one time with the SC1 kinetic constant set to zero, and a second time with the SC1 kinetic constant set to its nominal value, where the corresponding catalytic currents are subtracted.

\subsection{Boundary conditions}

The boundary conditions for $M_{\text {red }}$ and $Y_{\mathrm{ox}}$ at the electrode surface are shown in eqn (7) and (8). $Z_{\mathrm{ox}}$ cannot undergo electron transfer at the electrode surface; therefore, it has a "no-flux" boundary condition. 
The boundary condition at the film/electrolyte interface is different depending on the species involved. Since the mediator is immobilized in the film, the intermediate boundary condition for $M_{\text {red }}$ is "no-flux", which requires that the concentration gradient be equal to zero at the film/electrolyte interface when approaching the film boundary from domain I on the left side $\left(l_{1}{ }^{-}\right)$, as shown in eqn (9). The boundary condition for $M_{\text {red }}$ in the bulk is that its concentration is zero, the same value that it has in the surrounding solution domain.

$$
\left(\frac{\partial M_{\mathrm{red}}}{\partial x}\right)_{x=l_{1}^{-}}^{\mathrm{I}}=0
$$

Since $Y_{\text {red }} \mid Y_{\text {ox }}$ is freely diffusing throughout the system, in particular, between the film and the surrounding solution, and without mass transfer resistance, the intermediate boundary condition for $Y_{\mathrm{ox}}$ is "perfect-flux", where the concentration gradients just before and just after the film/solution interface are equal, as shown in eqn (10).

$$
\left(\frac{\partial Y_{\mathrm{ox}}}{\partial x}\right)_{x=l_{1}^{-}}^{\mathrm{I}}=\left(\frac{\partial Y_{\mathrm{ox}}}{\partial x}\right)_{x=l_{1}{ }^{+}}^{\mathrm{II}}
$$

The bulk semi-infinite boundary condition holds for $Y_{\mathrm{ox}}$ and $Z_{\mathrm{ox}}$, therefore, their concentrations at the bulk must remain undisturbed at their initial values, and with a slope of zero. At the end of each simulation, the concentration profiles at the end time are inspected, and if necessary, the simulation is repeated with a greater distance.

\section{Dimensionless groups}

After scaling of the major variables with respect to their maximum possible values, the dimensional parameters were formed into dimensionless groups. Two steps were deliberately taken in an effort to simplify and to unify the treatment of the dimensionless groups: (1) classification of the groups into "types", where the respective rate ratios are as transparent as possible, and (2) the use of double script notation, where it is easily seen which rates are being compared.

Inspection of the scaled main equations and the scaled electrode surface boundary conditions shows three main kinds of dimensionless groups: " $k$ " type groups, “ $\omega$ ” type groups, and " $\sigma$ " type groups. As was done in a reference model, ${ }^{19}$ various $\kappa$ type groups are used related to the various reaction-diffusion processes, which occur within the volume of the film or in the surrounding solution. In the model, single script notation was used to denote the particular chemical process of interest. The use of $\omega$ groups was inspired from another reference model, ${ }^{20}$ which focuses on transient electron transfer within redox-active films. Finally, $\sigma$ type groups denote electron transfer processes at an electrode surface. One example of each of these group types is described in detail in the following sections. A summary of the dimensionless groups in the model is included in the ESI. $\dagger$ 


\section{1 $\kappa_{\mathrm{SC} 1}^{\mathrm{Y}}$ as an example of a $\kappa$ type group}

The $\kappa_{\mathrm{SC} 1}^{\mathrm{Y}}$ group is shown in its most simplified form in eqn (11). The similarity of this expression to $\lambda / \sigma^{2}$ in a reference model, ${ }^{17}$ and to $\kappa$ in another reference model ${ }^{18}$ were helpful for interpreting this new dimensionless group as the ratio of the rate of SC1 to the rate of diffusion of Y.

$$
\kappa_{\mathrm{SC} 1}^{\mathrm{Y}}=\frac{l_{1}^{2} k_{\mathrm{SC} 1} M_{\mathrm{tot}}}{D_{\mathrm{Y}}}
$$

This ratio can be more clearly demonstrated after multiplication of the numerator and denominator by $Y_{\text {tot }}$ and rearrangement; the result is shown in eqn (12). In this equation, the units of the numerator and the denominator are $\mathrm{mol} \mathrm{cm} \mathrm{cm}^{-3} \mathrm{~s}^{-1}$; the numerator therefore represents the maximum possible rate of SC1 (when both reaction species are at their maximum possible concentrations), and the denominator represents the maximum molar diffusion rate of SC1 in a basis area of $l_{1}^{2}$.

$$
\kappa_{\mathrm{SC} 1}^{\mathrm{Y}}=\frac{k_{\mathrm{SC} 1} Y_{\mathrm{tot}} M_{\mathrm{tot}}}{\left[\begin{array}{ll}
\left(D_{Y} Y_{T o t}\right) / l_{1}^{2}
\end{array}\right]} \propto \frac{\mathrm{SC} 1 \text { reaction rate }}{\mathrm{Y} \text { diffusion rate }}
$$

The inverse square root of $\kappa_{\mathrm{SC} 1}^{\mathrm{Y}}$, which is shown in eqn (13), is also of interest because it allows for one to think of the same process in terms of the SC1 reaction layer.

$$
\left(\kappa_{\mathrm{SC} 1}^{\mathrm{Y}}\right)^{-1 / 2}=\frac{\sqrt{D_{\mathrm{Y}}\left(k_{\mathrm{SC} 1} M_{\mathrm{tot}}\right)^{-1}}}{l_{1}} \propto \frac{\mathrm{SC} 1 \text { reaction layer }}{\text { film thickness }}
$$

The reaction layer concept, which was introduced and emphasized in a foundational reference model, ${ }^{17}$ and was also used in a later reference model $^{18}$ was useful for the interpretation of this dimensionless group as the fractional distance in the film that a formed $Y_{\text {red }}$ molecule will be able to travel within the film before undergoing SC1, ignoring all other processes in the system.

\section{2 $\omega_{\mathrm{Y}}$ as an example of a $\omega$ type group}

The $\omega_{\mathrm{Y}}$ group is shown in its simplest form in eqn (14). It can be rearranged as a ratio of two time scales, as shown in eqn (15). In this form, $\omega_{\mathrm{Y}}$ can be interpreted as the ratio of the minimum time needed for a film of a basis area of $l_{1}^{2}$ to be fully saturated with $\mathrm{Y}$ by diffusion versus the exposure time. As such, $\omega_{\mathrm{Y}}$ is an indicator of how much of the film is accessible to $\mathrm{Y}$ by diffusion at the given experimental time scale.

$$
\begin{gathered}
\omega_{\mathrm{Y}}=\frac{l_{1}^{2}}{D_{\mathrm{Y}} t_{\exp }} \\
\omega_{\mathrm{Y}}=\frac{\left(l_{1}^{2} / D_{\mathrm{Y}}\right)}{t_{\exp }} \propto \frac{\text { minimum diffusion time }}{\text { experimental time scale }}
\end{gathered}
$$


The inverse square root of $\omega_{\mathrm{Y}}$, which is shown in eqn (16), is also of interest because it allows for one to think of the same process in terms of the Y diffusion layer.

$$
\left(\omega_{\mathrm{Y}}\right)^{-1 / 2}=\frac{\sqrt{D_{\mathrm{Y}}\left(t_{\exp }\right)}}{l_{1}} \propto \frac{\mathrm{Y} \text { diffusion layer }}{\text { film thickness }}
$$

Comparison to the $w$ dimensionless group in a reference mode $\mathbf{l}^{\mathbf{2 0}}$ was helpful for the interpretation of this new dimensionless group as the fractional distance in the film that a $Y_{\mathrm{ox}}$ molecule will be able to travel within the film in the time given, ignoring all other processes in the system.

\section{3 $\sigma_{\mathrm{SC} 2}^{\mathrm{Y}}$ as an example of a $\sigma$ type group}

The $\sigma_{\mathrm{SC} 2}^{\mathrm{Y}}$ group arises from the non-dimensionalization of the Butler-Volmer equation, and therefore is composed of dimensionless groups related to $k_{\mathrm{Y}}^{0}\left(v_{\mathrm{Y}}\right.$, shown in eqn (17)), the overpotential ( $\varepsilon_{\mathrm{Y}}$, shown in eqn (18)), and to $\alpha_{\mathrm{Y}}$ (the apparent electron transfer coefficient).

$v_{\mathrm{Y}}$ is the only factor in $\sigma_{\mathrm{SC} 2}^{\mathrm{Y}}$ that is outside of an exponent in eqn (19), therefore the units of this term will determine the overall units of $\sigma_{\mathrm{SC} 2}^{\mathrm{Y}}$. Multiplication of the numerator and denominator of $v_{\mathrm{Y}}$ by $Y_{\text {tot }}$ and rearrangement results in units of $\mathrm{mol} \mathrm{cm} \mathrm{cm}^{-2} \mathrm{~s}^{-1}$ in the numerator and the denominator. This allows for the interpretation of this dimensionless group as the rate of heterogeneous electron transfer of $\mathrm{Y}$ at the electrode surface versus the rate of diffusion of a surface plane of $\mathrm{Y}$, without considerations related to overpotential and the apparent electron transfer coefficient.

$$
\begin{gathered}
v_{\mathrm{Y}}=\frac{l_{1} k_{\mathrm{Y}}^{0}}{D_{\mathrm{Y}}}=\frac{k_{\mathrm{Y}}^{0} Y_{\mathrm{tot}}}{\left(D_{\mathrm{Y}} Y_{\mathrm{tot}} / l_{1}\right)} \\
\varepsilon_{\mathrm{Y}}=\frac{\left(E_{\mathrm{hold}}-E_{\mathrm{Y}}^{0}\right)}{\left(R T / z_{\mathrm{Y}} F\right)}
\end{gathered}
$$

In keeping with the notation from a reference textbook, ${ }^{21}$ a reduction at the electrode surface is considered as a "forward" reaction, and oxidation is conversely regarded as a "backwards" reaction. Since SC2 occurs through oxidation of $Y_{\text {red }}$, the "backwards" reaction is $\mathrm{SC} 2$; therefore $\sigma_{\mathrm{b}}^{\mathrm{Y}}$ can be regarded as $\sigma_{\mathrm{SC} 2}^{\mathrm{Y}}$ for this case as shown in eqn (19), and can be interpreted as the rate of SC2 versus the diffusion rate of $\mathrm{Y}$, which includes the effects of the applied overpotential and of the apparent electron transfer coefficient on the electron transfer rate.

$$
\sigma_{\mathrm{b}}^{\mathrm{Y}}=\sigma_{\mathrm{SC} 2}^{\mathrm{Y}}=v_{\mathrm{Y}} \exp \left[\varepsilon_{\mathrm{Y}}\left(1-\alpha_{\mathrm{Y}}\right)\right] \propto \frac{\mathrm{SC} 2 \text { reaction rate }}{\mathrm{Y} \text { diffusion rate }}
$$

\subsection{Secondary dimensionless groups: $\mu$ and $\theta_{\mathrm{MM}}$}

A secondary group, $\mu$, is shown in eqn (20). As the ratio of the Michaelis-Menten constant and the maximum substrate concentration, it denotes the fractional degree of enzyme saturation. 


$$
\mu=\frac{K_{\mathrm{M}}}{Y_{\mathrm{tot}}}
$$

$\theta_{\mathrm{MM}}$ is a secondary group because it can be represented as a ratio of two individual $\kappa$ groups. Starting from its simplified form, shown in eqn (21), multiplication of the numerator and the denominator by $P_{\text {tot }}$ demonstrates how $\theta_{\mathrm{MM}}$ can be interpreted as a ratio of the catalysis and electron transfer rates, as shown in eqn (22).

$$
\begin{gathered}
\theta_{\mathrm{MM}}=\frac{k_{\mathrm{cat}}}{k_{\mathrm{MP}} M_{\mathrm{Tot}}} \\
\theta_{\mathrm{MM}}=\frac{k_{\mathrm{cat}} P_{\mathrm{tot}}}{k_{\mathrm{MP}} M_{\mathrm{tot}} P_{\mathrm{tot}}} \propto \frac{\text { catalytic reaction rate }}{\text { electron transfer rate }}
\end{gathered}
$$

Since $\kappa$ groups are ratios of reaction and diffusion rates, $\kappa_{\text {cat }}^{\mathrm{Y}}$ and $\kappa_{\mathrm{MP}}^{\mathrm{Y}}$ can be defined by eqn (23) and (24).

$$
\begin{aligned}
\kappa_{\mathrm{cat}}^{\mathrm{Y}} & =\frac{k_{\mathrm{cat}} P_{\mathrm{tot}}}{\left(D_{\mathrm{Y}} Y_{\mathrm{tot}} / l_{1}^{2}\right)} \propto \frac{\text { catalytic reaction rate }}{\mathrm{Y} \text { diffusion rate }} \\
\kappa_{\mathrm{MP}}^{\mathrm{Y}} & =\frac{k_{\mathrm{MP}} M_{\mathrm{tot}} P_{\mathrm{tot}}}{\left(D_{\mathrm{Y}} Y_{\mathrm{tot}} / l_{1}^{2}\right)} \propto \frac{\text { electron transfer rate }}{\mathrm{Y} \text { diffusion rate }}
\end{aligned}
$$

Similarly, $\kappa_{\text {cat }}^{\mathrm{MP}}$ denotes the ratio of the catalytic reaction and electron transfer rates as shown in eqn (25).

$$
\kappa_{\text {cat }}^{\mathrm{MP}} \propto \frac{\text { catalytic reaction rate }}{\text { electron transfer rate }}
$$

$\kappa_{\text {cat }}^{\mathrm{MP}}$ can then be expressed as the ratio of $\kappa_{\text {cat }}^{\mathrm{Y}}$ and $\kappa_{\mathrm{MP}}^{\mathrm{Y}}$, which is equal to $\theta_{\mathrm{MM}}$, as shown in eqn (26).

$$
\kappa_{\mathrm{cat}}^{\mathrm{MP}}=\left(\frac{\kappa_{\mathrm{cat}}^{\mathrm{Y}}}{\kappa_{\mathrm{MP}}^{\mathrm{Y}}}\right)=\frac{k_{\mathrm{cat}} P_{\mathrm{tot}}}{k_{\mathrm{MP}} M_{\mathrm{tot}} P_{\mathrm{tot}}}=\theta_{\mathrm{MM}}
$$

\subsection{The SC1/SC2 ratio by the use of groups with double notation}

Double script notation can be useful for deriving expressions for rate comparisons of interest. For example, a dimensionless expression which is indicative of the relative rates of $\mathrm{SC} 1$ in the film and SC2 at the electrode surface can be derived, for the case where the oxidation of $Y_{\text {red }}$ is strongly favored. This can be calculated from the ratio of $\kappa_{\mathrm{SC} 1}^{\mathrm{Y}}$ (eqn (12)) and $\sigma_{\mathrm{SC} 2}^{\mathrm{Y}}$ (eqn (19)), in which the Y diffusion rate cancels out; the final result is shown in eqn (27).

$$
\frac{\kappa_{\mathrm{SC} 1}^{\mathrm{Y}}}{\sigma_{\mathrm{SC} 2}^{\mathrm{Y}}}=\frac{l_{1} k_{\mathrm{SC} 1} M_{\mathrm{tot}}}{k_{\mathrm{Y}}^{0} \exp \left[\varepsilon_{\mathrm{Y}}\left(1-\alpha_{\mathrm{Y}}\right)\right]} \propto \frac{\mathrm{SC} 1 \text { reaction rate }}{\mathrm{SC} 2 \text { reaction rate }}
$$


The appearance of $l_{1}$ in the numerator of eqn (27) implies that for an extremely high value of $l_{1}$ (extremely thick films), recombination is much more likely to be by SC1 than by SC2; this is physically reasonable since for very thick films, most of the $Y_{\text {red }}$ would be generated further away from the electrode. However, the likelihood of SC1 versus SC2 also depends on the relative kinetic parameters, as well as on the apparent electron transfer coefficient. For example, extremely high SC2 kinetics together with extremely low SC1 kinetics can therefore result in a higher likelihood for SC2 over SC1, even in a very thick film.

The derivation of expressions such as eqn (27) are useful because they are order of magnitude estimates of the individual rate ratios of interest. However, such expressions do not negate the need for full simulations, which include simultaneous considerations of all competing rates in the system, and which therefore generate exact results regarding the behavior of the system under a given set of conditions.

\section{Numerical solution of the modeling equations}

Because of the "no-flux" film/solution boundary condition for the reduced form of the mediator (eqn (9)), it was not possible to use built-in Matlab PDE solver functions, which ignore no-flux internal boundary conditions. Therefore, the space variable of the PDE system was discretized, resulting in a series of simultaneous first-order ordinary differential equations (ODEs) in time according to the method of lines. ${ }^{22}$ The discretization of the space variable was performed using a vertex-centered finite volume scheme with variable $\mathrm{x}$-spacing. ${ }^{22-24}$ Custom functions for single and double exponentially expanding grids were constructed, according to a method suitable for electrochemical simulations. ${ }^{25}$ The finite volume method was chosen due to its strengths related to spatial discontinuities (since it is an integral-based method), and to its strengths related to adherence to the conservation equations (since the governing equation is solved in "conservative" form). The finite volume method schematic and the finite difference equations are included in the ESI. $\dagger$

The system of ODEs was then solved using a Matlab built-in ordinary differential equation solver (ode15s), which is designed specifically for systems in which concentration profiles increase steeply over short distances (i.e. numerically "stiff"22,26). The time discontinuities within the system (i.e. light on and off) were managed within ode15s by time-dependent coefficients which were changed by steep linear on/off ramps. Numerical solution of the system allowed for the calculation of a "deconvoluted" current-time curve, which shows all contributions (direct and indirect) to the observed total current, for calculated concentration profiles at specified times, and for the generation of concentration profile animations at all time points of the simulation.

After implementation of the simulation was completed, a series of calculations were performed in order to verify the correctness of the model. As much as possible, this verification was performed in a "piecewise" way, in which the model was simplified for direct and quantitative comparison to the results from relevant reference models. For example, for verification of the correct implementation of the heterogeneous electron transfer at the electrode by SC2, the kinetics for all chemical processes was set to zero, and the resulting current-time curve was compared to expected results from an analytical expression for quasi- and 
irreversible electron transfer in a potential step experiment. ${ }^{21}$ For verification of the SC1 process, reduction of the model was not possible. Therefore, in this case, a general material balance which simultaneously considers the initial and final concentration profiles as well as the corresponding current-time curve was used. More details and examples related to the piecewise verification are given in the ESI. $\dagger$

\section{Applications of the model}

Such a model is useful for its predictive power, especially for situations or conditions where the outcome is unclear with qualitative estimation. Due to the large number of parameters, as well as the uniqueness of each individual experimental system, it is useful if simulations can be run to explore how parameters affect the photocurrent generation. In order to facilitate this, a standalone app was developed (see ESI, $\uparrow$ Standalone App for Simulations).

As a case study we performed simulations to predict the effect of the diffusion coefficient of the charge carrier $\left(D_{\mathrm{Y}}\right)$ on photocurrent generation as a function of the kinetic constant for the recombination $\left(k_{\mathrm{SC} 1}\right)$ of the reduced charge carrier $Y_{\text {red }}$ with the electron mediator $M_{\mathrm{ox}}$. The schematic illustration of the reactions is shown in Fig. 3A. In this particular example, we model the reaction between the photosynthetic protein and the charge carrier by means of Michaelis-Menten kinetics. The recombination of $Y_{\text {red }}$ at the electrode (SC2) is set as zero to unambiguously reveal the effect of $D_{\mathrm{Y}}$ and $k_{\mathrm{SC} 1}$ on photocurrent generation. As highlighted in Fig. 3A, $D_{\mathrm{Y}}$ is involved in two competing processes. In the photocatalytic portion of the scheme, $D_{\mathrm{Y}}$ defines the flux of $Y_{\mathrm{ox}}$ to the photocatalytic reaction layer and thus an increase in $D_{\mathrm{Y}}$ would be expected to be beneficial to the photocatalytic process. However, $D_{\mathrm{Y}}$ also defines the flux of $Y_{\text {red }}$ to the reaction layer for recombination of $Y_{\text {red }}$ with $M_{\text {ox }}$, so an increase in $D_{\mathrm{Y}}$ leads to a faster recombination rate. Since the rate of the photocatalytic process and the rate of recombination have opposite effects on photocurrent generation, the impact of $D_{\mathrm{Y}}$ on the system cannot be predicted based on a qualitative comparison of the two processes. Instead, simulations are required in order to quantitatively predict the effect of $D_{\mathrm{Y}}$ on the photocurrent generation.

The simulations were performed for a set of parameters (see the ESI $\dagger$ ) that ensure that mass transport of $Y_{\mathrm{Ox}}$ is limiting the photocatalytic process. 77 current-time curves were generated for 7 different values of $D_{\mathrm{Y}}$ and 11 different values of $k_{\mathrm{SC} 1}$ while all other parameters where kept constant. Examples of deconvoluted current-time curves for increasing $k_{\mathrm{SC} 1}$ values are shown in Fig. 3B for $D_{\mathrm{Y}}=6 \times 10^{-6} \mathrm{~cm}^{2} \mathrm{~s}^{-1}$. For the lowest value of $k_{\mathrm{SC} 1}$ (below $10^{2} \mathrm{M}^{-1} \mathrm{~s}^{-1}$ ), a steady state photocurrent is obtained which is mostly overlaying with the predicted current for the one corresponding to $k_{\mathrm{SC} 1}$ set to $10^{1} \mathrm{M}^{-1} \mathrm{~s}^{-1}$. As $k_{\mathrm{SC} 1}$ is increased, the photocurrent-time curves increasingly deviate from the pure photocatalytic curve. At transition values for $k_{\mathrm{SC} 1}$ (for instance $10^{5} \mathrm{M}^{-1} \mathrm{~s}^{-1}$ and $10^{7}$ $\mathrm{M}^{-1} \mathrm{~s}^{-1}$ ) the photocathodic current is lower and decreases over time during illumination while an anodic current appears in the following dark phase. These features are characteristic for recombination processes. At the highest $k_{\mathrm{SC} 1}$ values (above $10^{9} \mathrm{M}^{-1} \mathrm{~s}^{-1}$ ), both the photocurrent and the dark current completely vanish. The same qualitative trend of photocurrent decrease with increasing $k_{\mathrm{SC} 1}$ is observed for all investigated values of $D_{\mathrm{Y}}$. 
A

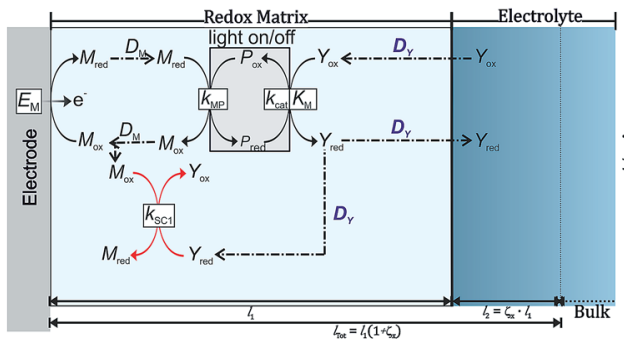

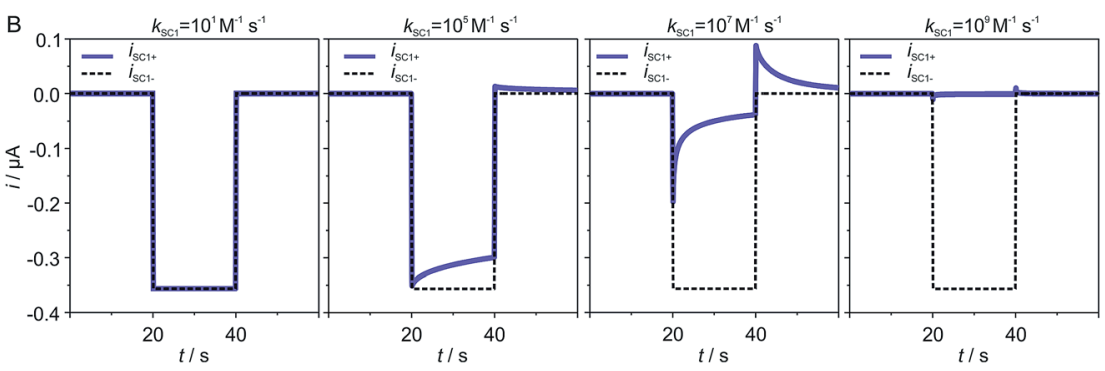

Fig. 3 (A) Schematic illustration of the reactions for the biophotocathode based on a photoenzyme generating a charge carrier that diffuses into the bulk of the electrolyte. The recombination pathway is limited to the reaction between the reduced charge carrier and the oxidized electron mediator. (B) Photocurrent predicted for the photocatalytic process alone (black dashed line) and for the recombination pathway associated to the photocatalytic process (blue solid line) for increasing values of $k_{\mathrm{SC} 1}$ at $D_{\mathrm{Y}}=6 \times 10^{-6} \mathrm{~cm}^{2}$ $s^{-1}$. (C) Photocurrent at $40 \mathrm{~s}$ (at the end of the illumination period) plot $v s$. $\log \left(k_{\mathrm{SC} 1}\right)$ for increasing values of $D_{Y}$.

Quantitative analysis of the impact of the diffusion coefficient is performed by plotting the photocurrent values (before the dark phase) against the $k_{\mathrm{SC} 1}$ values for each value of $D_{\mathrm{Y}}$ (Fig. 3C). The $i v s$. $k_{\mathrm{SC} 1}$ plots confirm the current cancelling effect of the charge recombination process irrespective of the value of $D_{\mathrm{Y}}$. However, the most important feature is that the transition in photocurrent loss in the $i v s . k_{\mathrm{SC} 1}$ curves is shifted to higher $k_{\mathrm{SC} 1}$ values as $D_{\mathrm{Y}}$ is increased. For a 10 -fold increase in $k_{\mathrm{SC} 1}$, a given value for the photocurrent can be maintained if $D_{\mathrm{Y}}$ is increased by a factor of 100 . These results demonstrate the ability to accommodate for increasing $k_{\mathrm{SC} 1}$ by increasing $D_{\mathrm{Y}}$ according to the relationship shown in eqn (28).

$$
i \propto \frac{\left(D_{\mathrm{Y}}\right)^{2}}{k_{\mathrm{SC} 1}}
$$

\section{Conclusions}

A model was developed for biophotoelectrodes based on photosynthetic proteins, in which the photocurrent can be simulated for the entire experiment, together with a deconvolution of the individual contributions to the total observed current, either by the catalytic process, or its loss through recombination processes within the redox film or at the electrode surface. Because of its strengths related to discontinuities in space (i.e. at the film/solution interface) and the balance of flux, 
the finite volume method within the context of the method of lines was used to solve the system of partial differential equations. The balance of flux is especially important for this problem because under stationary conditions, the substrate can diffuse in and out of the film. Besides photocurrents, time dependent concentration profiles were also predicted. Additionally, dimensionless groups which summarize the major processes in the system were developed and presented. The model is flexible and is therefore relevant for several possible systems with respect to the reaction between catalysts and the electron acceptor (modeled either as a bimolecular reaction, which is relevant for photosystem 1, or as a Michaelis-Menten enzymatic process, which is relevant for purple bacterial reaction centers). Similarly, the model can be adjusted either for direct generation of a charge carrier relevant for biophotovoltaic cells, or for an additional redox catalyst for the generation of a solar fuel as a final product.

The simulations were compiled into a stand-alone app, which can be used to investigate the effects of different parameters on photocurrent generation. The example given here, in which the effect of increasing charge carrier diffusion coefficient on the ability of the system to withstand increasing mediator-charge carrier recombination kinetics was investigated, shows the ability of the simulation to predict the performance of the system for complex situations where it is not possible by means of qualitative reasoning. The same simulation approach can be carried out for predicting the effects of any other parameters described in the model. Therefore, the model developed in this work will be helpful for the rational design and further optimization of biophotoelectrodes for maximal energy conversion efficiency.

\section{Author contributions}

All authors contributed in conceiving the research. D. B. supervised all modeling activities and developed the model on "charge carrier" generation. T. H. developed the model on "solar fuel" generation and the stand-alone app. H. Z. performed the electrochemical simulations. All authors contributed to writing the manuscript.

\section{Conflicts of interest}

There are no conflicts to declare.

\section{Acknowledgements}

This work was supported by the European Research Council (ERC Starting Grant 715900), by the DFG within the framework of the Cluster of Excellence RESOLV (EXC 1069), by the DFG/ANR within the projects SHIELD PL746/2-1 and No. ANR-15-CE050020. H. Z. is grateful for the support by the China Scholarship Council (CSC) (No. 201306950049). The authors thank Dierk Gruhn for helping with the simulations.

\section{Notes and references}

1 V. M. Friebe and R. N. Frese, Curr. Opin. Electrochem., 2017, 5, 126-134.

2 P. J. D. Janssen, M. D. Lambreva, N. Plumeré, C. Bartolucci, A. Antonacci, K. Buonasera, R. N. Frese, V. Scognamiglio and G. Rea, Front. Chem., 2014, 2, 36. 
3 K. Nguyen and B. D. Bruce, Biochim. Biophys. Acta, Bioenerg., 2014, 1837, 15531566.

4 T. Kothe, S. Pöller, F. Zhao, P. Fortgang, M. Rögner, W. Schuhmann and N. Plumeré, Chem.-Eur. J., 2014, 20, 11029-11034.

5 K. P. Sokol, D. Mersch, V. Hartmann, J. Z. Zhang, M. M. Nowaczyk, M. Rögner, A. Ruff, W. Schuhmann, N. Plumeré and E. Reisner, Energy Environ. Sci., 2016, 9, 3698-3709.

6 N. Plumeré and M. M. Nowaczyk, Biophotoelectrochemistry of Photosynthetic Proteins, in Biophotoelectrochemistry: From Bioelectrochemistry to Biophotovoltaics. Advances in Biochemical Engineering/Biotechnology, ed. L. Jeuken, Springer, Cham, 2016, 158, pp. 111-136.

7 V. Proux-Delrouyre, C. Demaille, W. Leibl, P. Sétif, H. Bottin and C. Bourdillon, J. Am. Chem. Soc., 2003, 125, 13686-13692.

8 A. F. Janzen and M. Seibert, Nature, 1980, 286, 584-585.

9 M. T. Robinson, D. E. Cliffel and G. K. Jennings, J. Phys. Chem. B, 2018, 122, 117-125.

10 P. N. Ciesielski, D. E. Cliffel and G. K. Jennings, J. Phys. Chem. A, 2011, 115, 3326-3334.

11 R. Caterino, R. Csiki, A. Lyuleeva, J. Pfisterer, M. Wiesinger, S. D. Janssens, K. Haenen, A. Cattani-Scholz, M. Stutzmann and J. A. Garrido, ACS Appl. Mater. Interfaces, 2015, 7, 8099-8107.

12 F. Milano, F. Ciriaco, M. Trotta, D. Chirizzi, V. de Leo, A. Agostiano, L. Valli, L. Giotta and M. R. Guascito, Electrochim. Acta, 2019, 293, 105-115.

13 G. Longatte, M. Guille-Collignon and F. Lemaître, ChemPhysChem, 2017, 18, 2643-2650.

14 G. Longatte, A. Sayegh, J. Delacotte, F. Rappaport, F. Wollman, M. GuilleCollignon and F. Lemaître, Chem. Sci., 2018, 9, 8271-8281.

15 O. Yehezkeli, R. Tel-Vered, J. Wasserman, A. Trifonov, D. Michaeli, R. Nechushtai and I. Willner, Nat. Commun., 2012, 3, 742.

16 K. R. Stieger, S. C. Feifel, H. Lokstein and F. Lisdat, Phys. Chem. Chem. Phys., 2014, 16, 15667-15674.

17 C. P. Andrieux, J. M. Dumas-Bouchiat and J. M. Savéant, J. Electroanal. Chem. Interfacial Electrochem., 1982, 131, 1-35.

18 P. N. Bartlett and K. Pratt, J. Electroanal. Chem., 1995, 397, 61-78.

19 V. Fourmond, S. Stapf, H. Li, D. Buesen, J. Birrell, O. Rüdiger, W. Lubitz, W. Schuhmann, N. Plumeré and C. Léger, J. Am. Chem. Soc., 2015, 137, 5494-5505.

20 K. Aoki, K. Tokuda and H. Matsuda, J. Electroanal. Chem. Interfacial Electrochem., 1983, 146, 417-424.

21 A. J. Bard and L. R. Faulkner, Electrochemical Methods: Fundamentals and Applications, Wiley, New York, 2nd edn, 2001.

22 M. E. Davis, Numerical Methods and Modeling for Chemical Engineers, Wiley, New York and Chichester, 1984.

23 V. Ruas, Numerical Methods for Partial Differential Equations: An Introduction Finite Differences, Finite Elements and Finite Volumes, John Wiley \& Sons, Chichester, West Sussex, United Kingdom, 2016.

24 W. J. Lick, Difference Equations From Differential Equations: Volume 41 of Lecture Notes in Engineering, Springer Verlag, 1989.

25 M. Rudolph, D. P. Reddy and S. W. Feldberg, Anal. Chem., 1994, 66, 589A-600A. 26 L. F. Shampine and M. W. Reichelt, SIAM J. Sci. Comput., 1997, 18, 1-22. 